\title{
A Network Experiment in Continuous Time: The Influence of Link Costs ${ }^{1}$
}

\author{
Siegfried K. Berninghaus ${ }^{2} \quad$ Karl-Martin Ehrhart ${ }^{3} \quad$ Marion Ott $^{4}$
}

March 2006

\footnotetext{
${ }^{1}$ We thank Sanjeev Goyal, Oliver Kirchkamp, Karim Sadrieh, Reinhard Selten, and Bodo Vogt for helpful comments. Financial support from the Deutsche Forschungsgemeinschaft, SFB 504, at the University of Mannheim, is gratefully acknowledged. NOTICE: this is the authors' version of a work that was accepted for publication in Experimental Economics. Changes resulting from the publishing process, such as peer review, editing, corrections, structural formatting, and other quality control mechanisms may not be reflected in this document. Changes may have been made to this work since it was submitted for publication. A definitive version was subsequently published in Experimental Economics (2006) 9:237-251, DOI 10.1007/s10683-006-9125-1.

${ }^{2}$ University of Karlsruhe, Institute WiOR, Zirkel 2, Rechenzentrum, D-76131 Karlsruhe (e-mail: berninghaus@wiwi.uni-karlsruhe.de).

${ }^{3}$ University of Karlsruhe, Institute WiOR, Zirkel 2, Rechenzentrum, D-76131 Karlsruhe (e-mail: ehrhart@wiwi.uni-karlsruhe.de).

${ }^{4}$ University of Karlsruhe, Institute WiOR, Zirkel 2, Rechenzentrum, D-76131 Karlsruhe (e-mail: ott@wiwi.uni-karlsruhe.de).
} 


\begin{abstract}
In recent work on non-cooperative network formation star-shaped networks play an important role. In a particular theoretical model of Bala and Goyal (2000) centersponsored stars are the only strict Nash networks. In testing this theoretical model, Falk and Kosfeld (2003) do not find experimental evidence that players select the centersponsored star. Based on a slight modification of Bala and Goyal's model, we design a network formation experiment in which, depending on link costs, periphery-sponsored stars and the empty network are the only strict Nash networks. We observe that almost all groups not only reach a strict Nash network once but also switch the center player in periphery-sponsored stars several times. The main innovation in our experiment is to use a continuous time framework which we believe to be a more realistic setting to study behavior in network formation situations and which makes coordination on stars much easier than simultaneous strategy adaptation in discrete time.
\end{abstract}

JEL classification: C72, C78, C92

Keywords: Network formation, Nash networks, real-time experiments 


\section{Introduction}

How people actually form and maintain (social) networks is an important behavioral topic which has various interdisciplinary aspects and which is addressed by sociologists, economists, physicists, computer scientists, and anthropologists. In our paper we elaborate the strategic behavior aspect of network formation. Strategic network formation deals with situations in which agents can build or sever costly links to other agents in a population to exchange valuable information. For recent overviews of different theoretical approaches see Jackson (2005) and Goyal (2005).

Our paper relates to the non-cooperative approach of network formation by Bala and Goyal (2000). In particular, we consider a variant of the so called two-way flow model which is characterized by information values flowing in both directions between two agents although only one agent bears communication costs. The linking decisions of agents are regarded as strategic decisions in a non-cooperative normal form game called network game. In network games, strict Nash networks are an appropriate equilibrium concept since the number of strict Nash networks is significantly smaller than the number of Nash networks. The empty network and the center-sponsored star are the only strict Nash networks of the network game of Bala and Goyal. In the center-sponsored star all connections are sponsored by one agent, called the center player. The empty network is a network without any link.

Our paper is inspired by recent experimental work of Deck and Johnson (2004), Callander and Plott (2005), and in particular by the results of Falk and Kosfeld (2003). The main intention of Falk and Kosfeld is to test the theoretical model by Bala and Goyal. As one of the most remarkable results of their experiments strict Nash networks are reached by many groups (about 50\%) only in the one-way flow framework while they are never reached in the two-way flow framework (the center-sponsored star). The authors offer some very interesting explanations for their experimental findings. To reach a center-sponsored star in the two-way flow model seems to be a complex task for an individual decision maker. One agent in the population has to build as many links as possible while the remaining players, even in a model in which building a single link generates positive net profits, build no links. This is a highly asymmetrical decision situation which can be dealt with by solving a complex coordination problem. Another explanation is based on fairness considerations. In the center-sponsored star network, the center player actually has a significantly lower equilibrium payoff than the periphery players since he subsidizes all connections in the network. Therefore, inequity aversion may be an issue in network formation. For an overview of experiments on network formation see Kosfeld (2004).

In our experiment, we apply a different design which allows only limited access to the information of neighbors. More precisely, it is an essential feature in our experiment to discriminate between actively reached, passively reached, and indirect neighbors. If a player $i$ opens a link to another player $j$ he has to pay for it. Player $j$ is then an actively reached neighbor of player $i$. On the other hand, player $i$ in this case is a passively reached neighbor of player $j$. It is essential in our experimental design that a player has access to the information values of his actively and passively reached 
neighbors and, furthermore, to his indirect neighbors, i.e. to those players that are actively or passively reached by his actively reached neighbors. Empirical investigations show that information will not flow between all neighbors on a path of arbitrary length in the network as it is assumed in the framework of Bala and Goyal (e.g. Friedkin 1983, Granovetter 1995). Note that this is also one of the main differences between our experimental design and the design of Falk and Kosfeld who suppose in accordance with the theoretical model by Bala and Goyal that a player can benefit from any other player who is reached via a finite number of links.1 In our particular framework, the so-called periphery-sponsored stan ${ }^{2}$ (ps-star) and, for some parameter range, the empty network are the only strict Nash networks.

The second main difference is that our experiment is conducted in continuous time. In this framework information is updated very often (approximately ten times per second). We suppose that the computer updates incoming information within very short time intervals that are smaller than players' reaction times. Thus, from an individual player's point of view the experiment seems to be conducted in continuous time. In our particular experimental design, subjects are allowed to select an action at any point in time within a fixed interval of thirty minutes. This slight modification of the standard simultaneous-choice discrete time design helps subjects to solve complex coordination tasks and, therefore, to coordinate easier on strict Nash networks.

We observe ps-stars for large time intervals in almost all groups. Moreover, in most groups several or all players are the center player in a ps-star for some time. Since payoffs in a ps-star are not equally distributed between center and periphery players inequity aversion seems to be the guiding motive of our subjects, because by switching the center players it is possible to approach an equalization of long run payoffs. This view is supported by the results of our experiment in which we analyze the impact of increasing link costs on network formation. Note that with increasing costs the payoffs differences in the ps-star grow. As a main result we observe an even higher intensity of changing ps-stars in groups with higher communication costs.

Note that in our continuous time framework the probability that more than one player changes his action at exactly the same point in time is nearly equal to zero. Thus, every player knows the network and therefore the action configuration on which he answers. Compared with simultaneous action adaptation in consecutive periods, this method has the advantages that a player does not have to build beliefs about the changes of the other players and that a player can decide himself on which network he wants to react. This is a different strategic situation for the players than in a framework with separate action adaptation (in predetermined or random order) in periods. Therefore, our experimental study offers the chance to gain insight into behavioral aspects of strategic network formation.

Another advantage of this method is that if a player realizes that he changed his action in a disadvantageous way he can immediately revoke the change and therefore

\footnotetext{
${ }^{1}$ Another way to at least reduce the influence of distant neighbors is to introduce decay (see Bala and Goyal 2000, Goeree, Riedl and Ule 2005).

${ }^{2}$ In a periphery-sponsored star all periphery players subsidize the connections of the center player who benefits from the information values of all other players without having to pay for it.
} 
the danger of far-reaching effects of a wrong decision on subsequent periods is greatly reduced.

Furthermore, we regard our experimental method in continuous time as more realistic and more suitable especially for experiments on network formation. People decide themselves when they want to contact others, when they want to build new relationships or ask for information. As in our framework they cannot decide when they are contacted by others but at least they decide when they are active themselves.

Our paper is organized as follows. In the next section, we present the theoretical model of a network game on which our experimental design in Section 3 is based. Sections 4 and 5 contain our experimental results and our analysis. Section 6 concludes.

\section{The network game}

The network game is characterized by the set of players $I=\{1, \ldots, n\}$, the strategy sets $G_{i}$, and payoff functions $\Pi_{i}$. An individual strategy of player $i$ is a vector $g_{i} \in G_{i}:=$ $\{0,1\}^{n}$ with the following interpretation. If $g_{i j}=1$ player $i$ establishes a link with player $j$, otherwise we have $g_{i j}=0$. By convention, a player cannot link with himself, that is $g_{i i}:=0$ for all $i \in I$. A player has to pay for each link that he establishes. Note that a link between two players in our model is supposed to be already established if at least one player wants to open it, i.e. if $\max \left\{g_{i j}, g_{j i}\right\}=1$ holds. If a link is established both players benefit from the exchange of information even if only one player has to pay for the connection (two-way flow model). Each strategy configuration $g=\left(g_{1}, \ldots, g_{n}\right)$ generates a directed graph denoted by $\mathcal{G}_{g}$, where the vertices represent players and a directed edge from $i$ to $j$ exists if $g_{i j}=1$.

In a network generated by the strategy configuration $g$ each player $i$ may have a number of agents with whom he is connected. We call these agents neighbors of $i$. It is essential for our model to distinguish three types of neighbors.

Actively reached neighbors are all players $i$ holds links with:

$$
N_{i}^{a}\left(g_{i}\right):=\left\{j \in I \mid g_{i j}=1\right\} .
$$

Links of $i$ with players in $N_{i}^{a}\left(g_{i}\right)$ are called $i$ 's active links or simply $i$ 's links.

The passively reached neighbors of $i$ are the players who hold a link with $i$ :

$$
N_{i}^{p}\left(\mathcal{G}_{g}\right):=\left\{j \in I \mid g_{j i}=1\right\} .
$$

A link of $j$ to $i$ is called a passive link of $i$.

We call indirect neighbors of $i$ all actively or passively reached neighbors of all actively reached neighbors of $i$, i.e.

$$
N_{i}^{\text {ind }}\left(\mathcal{G}_{g}\right):=\left\{k \in I \mid \exists j \neq i \neq k: g_{i j}=1 \text { and } \max \left\{g_{j k}, g_{k j}\right\}=1\right\} .
$$

Thus, the set of all neighbors of player $i$ is given by

$$
N_{i}\left(\mathcal{G}_{g}\right):=N_{i}^{a}\left(g_{i}\right) \cup N_{i}^{p}\left(\mathcal{G}_{g}\right) \cup N_{i}^{\text {ind }}\left(\mathcal{G}_{g}\right) .
$$


Let $n_{i}\left(\mathcal{G}_{g}\right)$ denote the number of elements in $N_{i}\left(\mathcal{G}_{g}\right)$ and $n_{i}^{a}\left(g_{i}\right)$ the number of elements in $N_{i}^{a}\left(g_{i}\right)$. Note that both actively and passively reached neighbors are direct neighbors (in contrast to indirect neighbors). That is, they are connected in the associated directed graph by a directed edge. Since direct neighbors in our model are treated differently depending on how the links with them are financed it makes sense to subdivide the class of direct neighbors into actively and passively reached ones.

Costs for opening a link are supposed to be the same for each player and are denoted by $c(>0)$. The benefit or return which player $i$ can extract from being connected (actively, passively, or indirectly) with player $j$ is the same for all players and supposed to be equal to $a(>0)$. Given strategy configuration $g=\left(g_{1}, \ldots, g_{n}\right)$, player $i$ 's payoff or net return is given by

$$
\Pi_{i}(g):=a n_{i}\left(\mathcal{G}_{g}\right)-c n_{i}^{a}\left(g_{i}\right) .
$$

We see from this definition that player $i$ may benefit from a connection to $j$ although he does not have to pay for it 3 We also find that player $i$ 's payoff is equal to zero if $i$ is an isolated player in the network, that is, if $N_{i}\left(\mathcal{G}_{g}\right)$ is an empty set.

In contrast to our model Bala and Goyal (2000) assume that the set of neighbors of player $i$ consists of all players connected with $i$ by a path in the non-directed graph associated with $\mathcal{G}_{g}$.

We believe that our neighborhood concept is certainly more realistic for real-world networks than the assumption that players have access to the information of players who are connected via a path of arbitrary length in the network. In labor markets, for example, social networks play a significant role in getting a job. In the empirical investigation of Granovetter (1995) the main part of the people, who got their job via social contacts, had heard about it from their friends, relatives or acquaintances $(39 \%$ of the cases), or from acquaintances of those (45\%). That is, from a network point of view the person who is looking for a job has access to the information of his "neighbors" who are at the most two "steps" away. Furthermore, $48 \%$ of those who had heard about their job from their friends were looking for a job, $52 \%$ had heard of their current job through friends but were not looking for a new job. Finally, $72 \%$ of those employees who had received the information about their job from friends of friends were looking for jobs. This supports our interpretation of indirect connections that are only accessible via active links 5

For network games $\Gamma=\left\{G_{1}, \ldots, G_{n} ; \Pi_{1}(\cdot), \ldots, \Pi_{n}(\cdot) ; I\right\}$ a Nash equilibrium is a matrix of individual link decision vectors $g^{*}=\left(g_{1}^{*}, \ldots, g_{n}^{*}\right)$ such that no single player $i$ has an incentive to open additional links than given by $g_{i}^{*}$ or to sever links prescribed by $g_{i}^{*}$.

\footnotetext{
${ }^{3}$ For example, if $g_{i j}=0$, but $g_{j i}=1$ holds.

${ }^{4}$ This assumption contrasts with the framework of Bala and Goyal in which a player receives a payoff of $a$ even if he is isolated in the network.

${ }^{5} \mathrm{McBride}$ (2006) provides another motivation for deviating from the original Bala/Goyal model of payoff flows. Because of imperfect monitoring it might be difficult to consider links to agents who are far away.
} 
Definition 1 A strategy configuration $g^{*}$ in $\Gamma$ is a Nash equilibrium if

$$
\text { for all } i: \quad \Pi_{i}\left(g_{-i}^{*}, g_{i}^{*}\right) \geq \Pi_{i}\left(g_{-i}^{*}, g_{i}\right) \text { for all } g_{i} \in G_{i},
$$

where $g_{-i}^{*}=\left(g_{1}^{*}, \ldots, g_{i-1}^{*}, g_{i+1}^{*}, \ldots, g_{n}^{*}\right) . \mathcal{G}_{g^{*}}$ is the Nash network generated by $g^{*}$.

Moreover, if in (1) the strict inequality holds for all $i$ and $g_{i}$, the strategy configuration $g^{*}$ is called a strict Nash equilibrium. ${ }^{6}$

By altering the model of Bala and Goyal their theoretical results do no longer hold. For example, the center-sponsored star, a strict Nash network in their two-way flow model, is not even a Nash network in our modified framework. We calculated all Nash networks for the parameter values of our experiment. The results of our analysis for benefits $a=3$ and low costs of $c=2$ are summarized in Berninghaus, Ehrhart, Ott and Vogt (2004). Because of the large number of Nash networks in this case, strict Nash networks are a reasonable refinement.7 In games with link costs $c>a$, no weak Nash networks exist. Depending on the particular parameter constellation in our experiment strict Nash networks can be characterized as periphery-sponsored stars and/or the empty network. In the following we give conditions for a periphery-sponsored star and the empty network being a strict Nash network in our framework.

Proposition 1 If $n>3$ strict Nash architecture $\$^{8}$ are:

(a) for $c \leq a$ the periphery-sponsored star (ps-star),

(b) for $a<c<(n-1)$ a the ps-star and the empty network,

(c) for $(n-1) a \leq c$ the empty network.

For the proof see Berninghaus, Ehrhart and Ott (2005).

We call a network efficient when the sum of individual payoffs is maximized. Note, if $c<n a$ the ps-star is the only efficient network? while the empty network is the only efficient network for $c>n a$. If $c=n a$ both are efficient.

\section{Experimental design}

The computerized experiment was conducted in the experimental laboratory at the University of Karlsruhe. Subjects were randomly selected from a pool of students of various faculties. The experiment was organized in 14 sessions with two groups of six

\footnotetext{
${ }^{6} \mathrm{~A}$ network which is generated by a strict or a non-strict Nash equilibrium is called a strict Nash network or a weak Nash network, respectively.

${ }^{7}$ For $a=3$ and $c=2,3666$ Nash networks exist. Of those, six are strict Nash networks.

${ }^{8}$ Two networks have the same architecture if one network can be obtained from the other by permuting the labels of agents. For example for $n=6$ players the ps-star architecture has 6 configurations, i.e. the ps-stars are 6 of $\left(2^{5}\right)^{6}=1,073,741,824$ possible networks or 1 of 1,540,944 possible architectures (Oberschelp 1967).

${ }^{9}$ In a ps-star each player extracts the maximum value from the population with a minimum of total link costs.
} 


\begin{tabular}{lllll}
\hline & Treatment I & Treatment II & Treatment III & Treatment IV \\
\hline $\begin{array}{l}\text { Strict Nash } \\
\text { equilibrium }\end{array}$ & ps-star & $\begin{array}{l}\text { ps-star and } \\
\text { empty network }\end{array}$ & $\begin{array}{l}\text { ps-star and } \\
\text { empty network }\end{array}$ & empty network \\
Efficient network & ps-star & ps-star & ps-star & ps-star \\
Link costs $c$ & 2 & 7 & 13 & 16 \\
[ExCU $/$ minute $]$ & & & & 4 \\
Number of groups & 8 & 8 & 8 & 4 \\
\hline
\end{tabular}

Table 1: Characterization of treatments

subjects participating in each session. The software for the experiment was developed in our laboratory to conduct experiments in continuous time.

In our setting the network game as described in Section 2 is repeated in continuous time. Each game lasts 30 minutes. The game starts when all subjects have made their first decision, i.e. each subject has to decide for every possible active link whether this link is built or not. Thereafter, subjects can change their actions, i.e. either open or sever links, at any time. Information is updated by the computer ten times per second. Particularly, the current payoff flow is computed every tenth of a second and accumulated payoff is "integrated" up to the given moment. The information presented on every subject's computer screen throughout the game includes the elapsed time, her current payoff flow and her current accumulated payoff. A subject's own and the other subjects' active links are illustrated graphically on the screen by arrows in a graph. Moreover, the subjects with whom a player is connected have a different color on her screen than the remaining ones (for details see the experimental instructions in Berninghaus et al. 2005).

The return per connected player is set equal to $a=3$ ExCU per minute (ExCU $=$ experimental currency units). The treatments differ in the costs per link. In Treatment I we set $c=2$ ExCU per minute which still guarantees a positive net return for each individual connection. The only strict Nash network architecture in Treatment I is the ps-star.10 In Treatments II, III, and IV we set $c=7, c=13$, and $c=16$ ExCU per minute, respectively. In these treatments opening a new link with another player who has no active or passive links is not profitable. Nevertheless, in Treatments II and III ps-stars and the empty network are strict Nash, while in Treatment IV only the empty network is a strict Nash network (see Proposition 1). An overview of the benchmark solutions of the base game is given in Table 1 .

Eight groups in each of the Treatments I-III give us eight independent observations for these treatments. We restrict the number of participating groups in Treatment IV to four, since the experimental results of these groups are unambiguous.

The payoff for each subject is accumulated over 30 minutes and paid out in cash after the experiment. For example, suppose a player extracts a payoff of 4 ExCU per

\footnotetext{
${ }^{10}$ There exist eleven Nash network architectures for Treatment I, but the time spent in weak Nash networks is negligible (for details see Berninghaus et al. 2005). In the other treatments only strict Nash networks exist.
} 
minute from the network for 15 seconds and then switches her action and obtains 2 ExCU per minute for 54 seconds. Her accumulated payoff for these 69 seconds is equal to $(4 \mathrm{ExCU} / 60) * 15+(2 \mathrm{ExCU} / 60) * 54=2.8 \mathrm{ExCU}$. The maximum and minimum payoff earned in the experiment is equal to $17.89 €$ and $12.20 €$, respectively $(14.83 €$ on average). Since subjects in Treatments II-IV have a lower expected payoff than subjects in Treatment I they get a show-up fee of $125 \mathrm{ExCU}$ and we vary the conversion rate.11

Before the experiment starts, subjects have to solve some selected problems on building links in a network and calculating the resulting payoff. Here, we try to assure that subjects do not become familiar with ps-stars and their characteristics.

\section{Experimental results}

It is one of the main features of our results that almost all groups (except for groups in Treatment IV) reach several ps-stars and, moreover, keep these networks for a considerable period of time. However, there are still significant differences in the results between the treatments which will be demonstrated in more detail below. More detailed group results can be found in Berninghaus et al. (2005).

\subsection{Results of Treatments I-IV}

Table 1 shows that the ps-star is an important benchmark for all treatments. As one of the main results we obtain that in Treatments I-III all groups but one (in Treatment I) reach the ps-star. Moreover, all but two of these groups (one in Treatment I, one in Treatment III) leave a ps-star (i.e. a strict Nash network) in order to form other ps-stars with different center players. In Treatments I, II, and III one, four, and five groups reach all six possible ps-stars, respectively. The minimum number of different ps-stars reached by a group was equal to zero, three, and one, respectively.

The average payoff does not vary much across the groups in all treatments. However, group behavior is not homogenous with respect to other aspects. For example, group 7 in Treatment I builds 21 ps-stars with six different center players while group 3 visits the same ps-star nine times. Similar variations can be found in the time the groups need to reach a ps-star for the first time. Group 4 needs $70 \%$ of the total time while group 3 needs only $11 \%$ of the total time to reach the first ps-star. In contrast, group 8 does not even reach one ps-star during the course of the experiment. Variations like this are also observed in Treatments II and III.

In Table 2 we compare Treatments I-III with respect to some selected performance criteria. In continuous time experiments, subjects typically change actions very often (e.g. Berninghaus, Ehrhart and Keser 1999, Berninghaus and Ehrhart 1998, 2003). Therefore, we consider the performance variable "number of action changes." As further

\footnotetext{
${ }^{11}$ The conversion rate is $1 €$ per 24 ExCU in Treatments I and II, $1 €$ per 15 ExCU in Treatment III, and $1 €$ per 10.5 ExCU in Treatment IV. The show-up fee is $0 \mathrm{ExCU}$ in Treatment I and $125 \mathrm{ExCU}$ in Treatments II-IV. The conversion rates are determined such that the sum of payoffs (in Euro) is the same in all treatments provided that the ps-star is played the whole time.
} 


\begin{tabular}{lccc}
\hline & Treatment I & Treatment II & Treatment III \\
\hline Avg. number of action changes & 352 & 444 & 357 \\
Avg. number of different networks formed & 306 & 388 & 213 \\
Avg. number of links in a group & 7 & 5 & 3 \\
\hline Number of groups with ps-stars & 7 & 8 & 8 \\
Avg. number of ps-stars & 10 & 16 & 14 \\
Avg. number of different ps-stars & 3 & 5 & 5 \\
Avg. time spent in ps-stars: & & & \\
$\quad$ Minutes (Percentage of total time [\%]) & $8(25)$ & $11(35)$ & $8(26)$ \\
Avg. time spent in a ps-star [seconds] & 45 & 41 & 33 \\
Avg. time until first ps-star reached: & & & \\
$\quad$ Minutes (Percentage of total time [\%]) & $12(40)$ & $3(9)$ & $6(20)$ \\
Avg. number of action changes until first ps- & 149 & 29 & 64 \\
star reached & & & 8 \\
\hline Number of groups with empty networks & 0 & 2 & 32 \\
Avg. number of empty networks & 0 & 1 & $6(21)$ \\
Avg. time spent in empty network: & & & 12 \\
$\quad$ Minutes (Percentage of total time [\%]) & $0(0)$ & $0(0)$ & 9.4 \\
Avg. time spent in an empty nw. [seconds] & - & 2 & 12.0 \\
\hline Avg. payoff [€] & 14.8 & &
\end{tabular}

Table 2: Comparison of Treatments I, II, and III

variables characterizing group performance we consider the number of different networks formed, the number of active links, the number of ps-stars and empty networks reached, the number of different ps-stars (i.e. ps-stars with different center players) the total time spent in ps-stars and empty networks, the average time spent in a ps-star and an empty network, the time and the number of action changes until the first ps-star is reached, and the average payoff per player. In Treatments II and III we consider performance with respect to the empty network which does not play any role in Treatment I.

Compared with the results in Treatment I, the average number of action changes is higher in Treatment II. In Treatment III as in Treatment I, subjects change strategies very often. However, the average number of changes is not as high as in Treatment II.

The time spent on average in a ps-star is 45, 41, and 33 seconds in Treatments I, II, and III, respectively. We consider these periods as long enough to interpret the psstar as rather stable in our continuous time framework. Although no player should be interested in a unilateral deviation from a ps-star we would not expect that subjects, once the group reached a ps-star, do nothing for the remaining 23 minutes on average. Facts in favor of our interpretation are that compared with the observed periods of time in other networks the 33 to 45 seconds are rather long and that very often ps-stars are left to build new ps-stars with other center players. Plausible reasons for the last 
argument will be explained in detail later (see Section 5.3).

Treatments I and II show differences in the results with respect to ps-stars. In Treatment II we observe more ps-stars that are played for a longer fraction of the total time. Moreover, the first ps-star is on average reached faster and with fewer action changes in Treatment II. How can we explain these differences? In Treatment II there seems to be greater pressure on the players to change the respective center player (i.e. to switch from one ps-star to the next) than in Treatment I. The payoff difference between center and periphery player in a ps-star is much larger in Treatment II than in Treatment I. The inequity aversion motive seems to put strong pressure on the players to change ps-stars so that each group member at least for some minutes has the chance to be in the advantageous position of a center player (see also Section 5.3). Otherwise, it would be difficult to reach an equalization of payoffs between all players in the long run. Furthermore, the increase in costs might induce the desire for more indirect connections when building an active link and therefore lead faster to the first ps-star. This is supported by the observation that in Treatment II on average fewer links exist than in Treatment I.

The empty network is never observed in Treatment I. Table 2 shows that in Treatment II only two groups reach the empty network and stay in it for a negligible fraction of the total time. Subjects seem to prefer efficient Nash networks. Another explanation for this behavior may be that there is a "natural resistance" of subjects to remain passive in an experimental environment, i.e. to opening no links at all.

On average the groups in Treatments II and III reach ps-stars with five different center players, i.e. almost every player is the center player for at least some time. In Treatment I we observe on average three different center players. This might be the result of the increased "pressure" to interchange center players in order to equalize profits in the long run in Treatments II and III since the payoff difference between center player and periphery players is significantly larger. As the number of different ps-stars reached is already very high in Treatment II an increase in Treatment III cannot be expected.

The theoretical benchmark is the same for Treatments II and III. In Treatment III we observe that, compared with Treatment II, on average fewer ps-stars are reached during the experiment. The time spent in a ps-star is lower than in Treatment II and the time until the first ps-star is reached is higher. Redundant links are punished more severely in Treatment III than in Treatment II which may favor subjects who rather stay in empty networks than open non-profitable links.

In contrast to Treatment II, groups in Treatment III spend significantly more time in the empty network. The average time of all groups spent in the empty network is about $21 \%$ of the total time. We could say that the pressure on the groups to switch ps-stars is dampened by the tendency to play empty networks.

As a remarkable fact of our experimental results, we emphasize that in Treatments II and III one and two groups, respectively, reach all different ps-stars by interchanging the center player three times in the same order. We might see this as coordinated rotation of center players where subjects use time and position as coordination device ${ }^{12} \mathrm{We}$

\footnotetext{
${ }^{12}$ In the experiment, for every subject the label of the other subjects on the screen is different, but the sequence of the subjects that are allocated on a circle is the same.
} 
consider this behavior in Treatments II and III as a strong argument in favor of inequity aversion.

The average group payoff is declining from Treatment I to Treatment II. This is easy to understand since redundant links are punished more seriously in Treatment II than in Treatment I. Due to the different calculation the payoff in Treatment III is not directly comparable with that of the other two treatments. However, it does not surprise that the payoff is lower as it is much harder to generate positive payoff flows in Treatment III. In fact, two subjects finish the experiment with pecuniary losses.

We omit a detailed presentation of the results of Treatment IV since the strategic decision problem is much more transparent than in the other treatments. In Treatment IV, the ps-star is not a strict Nash network but it is efficient. Because of the extremely high link costs we do not expect ps-stars to be formed at all during the course of the experiment. Indeed, the experimental results show that the groups spend most of the time in the empty network ( $67 \%$ of the total time) whereas no ps-star was reached at all. Most subjects do not hold any active link for a significant amount of time. As an extreme example we mention one particular group that played the empty network for $95 \%$ of the total time.

\subsection{The phenomenon of "fair stars"}

Group 8 in Treatment I is the only group in this treatment that does not reach a ps-star. A closer look shows that the subjects in this group for almost half of the total time get stuck in a network near a ps-star. This network is characterized as a ps-star in which the center player nullifies his payoff advantage by opening exactly one active link to one periphery player. This results in payoff equalization among the group members. We call this type of network a "fair star" in order to indicate that the center player seems to be motivated by establishing equal distribution of payoffs in the group. Note that fair star networks are neither Nash nor efficient. Group 8 stays in the fair star network about $43 \%$ of the whole time. It is remarkable that no observed ps-star (with a higher payoff for the center player but the same payoff for the periphery players) in any other group is as stable as this fair star. Fair star networks are not only formed in this group, we observe fair stars for short time periods in almost all groups (see Table 3).

\begin{tabular}{lccc}
\hline & Treatment I & Treatment II & Treatment III \\
\hline Number of groups with fair stars & 8 & 7 & 7 \\
Avg. number of fair stars & 6 & 3 & 5 \\
Avg. number of different center players & 2 & 2 & 2 \\
Avg. time spent in fair stars: & & & \\
Minutes (Percentage of total time [\%]) & $3(11)$ & $1(2)$ & $1(3)$ \\
Avg. time spent in a fair star [seconds] & 46 & 12 & 11 \\
\hline
\end{tabular}

Table 3: Comparison of Treatments I, II, and III with respect to fair stars

For three other groups in Treatment I such networks also prove to be quite attractive 
as they stay in fair stars for more than three minutes. On average, groups in Treatment I spend about $11 \%$ of the total time in fair stars. The situation changes in the other treatments. In Treatment IV, no fair stars are reached at all. In Treatments II and III fair stars are not as important as in Treatment I. The number of groups with fair stars and the number of different fair stars is similar, but the average percentage of time spent in fair stars is much lower as we observe fewer fair stars and those for shorter time intervals. The average percentage of time in the fair star is equal to $2 \%(3 \%)$ in Treatment II (III). The maximum time spent in fair stars per group is equal to $7 \%(8 \%)$. A center player forgoes 7 (13) ExCU per minute when forming a fair star instead of a ps-star in Treatment II (III) and only 2 ExCU in Treatment I. This may explain why fair stars play a more important role in Treatment I than in the other treatments.

Note, that for the stability of the fair star not only the behavior of the center player is important but also the periphery players' behavior. Every periphery player receives a payoff flow of $(n-1) a-c$ in the ps-star. In Treatments I and II this is higher than the payoff a periphery player can get when he deviates. However, in Treatment III unilateral deviation from the fair star is not only profitable for the center player but also for one periphery player. If the periphery player with the passive link from the center player deletes his only active link in the fair star his payoff flow increases from 2 to $3 \mathrm{ExCU}$ per minute.

We regard fair stars as one of several possible expressions of inequity aversion. In order to deepen this aspect of our experiment we analyze the transition behavior of groups to and from a fair star by looking at the action configuration one step before and one step after a fair star, respectively. If the action configuration one step before the fair star is a ps-star and the center player opens an additional link we call this a positive transition from a ps-star to a fair star. On the other hand, when a center player in a fair star severs the only link he holds with a periphery player we call this a negative transition from a fair star to a ps-star. By counting for each group the negative and positive transitions between ps-stars and fair stars we find that in Treatments I, II, and III of all fair stars

- 44\%, $50 \%$, and $60 \%$, respectively, are followed directly by a ps-star (negative transition),

- 4\%, $12 \%$, and $23 \%$, respectively, follow directly on a ps-star (positive transition),

- positive transitions are observed in one, two, and four groups, respectively.

If all transitions to fair stars were positive the phenomenon of fair stars would clearly indicate that center players prefer fair stars rather than ps-stars. However, our results on negative transitions show that fair stars are very often the last step on the way to a psstar. More positive transitions are observed in Treatments II and III than in Treatment I. This is not easy to understand since building positive transitions to fair stars is much more "expensive" than in Treatment I. However, one should not overemphasize these results since the total time spent in fair stars in Treatments II and III is still very low so that positive and negative transitions can be interpreted as the result of individual random choices. 


\section{Analysis of experimental results}

\subsection{Correlations}

In this section, we analyze correlations between some selected variables which are described in Table 2; number of action changes, number of different ps-stars reached, percentage of time spent in ps-stars, number of networks formed during the duration of the experiment, and the average per capita payoff. The results are presented in Table 4 where significant correlations based on a $5 \%$ significance level are presented in boldface.

\begin{tabular}{|c|c|c|c|c|c|c|c|c|}
\hline & \multirow[b]{2}{*}{ Variable 1} & \multirow[b]{2}{*}{ Variable 2} & \multicolumn{2}{|c|}{ Treatment I } & \multicolumn{2}{|c|}{ Treatment II } & \multicolumn{2}{|c|}{ Treatment III } \\
\hline & & & $r_{s}$ & ( $p$-value) & $r_{s}$ & ( $p$-value) & $r_{s}$ & ( $p$-value) \\
\hline 1) & $\begin{array}{l}\text { \# action } \\
\text { changes }\end{array}$ & $\begin{array}{l}\text { Time spent } \\
\text { in ps-stars }\end{array}$ & -0.76 & $(0.02)$ & -0.81 & $(0.01)$ & -0.19 & $(0.62)$ \\
\hline 2) & $\begin{array}{l}\text { \# action } \\
\text { changes }\end{array}$ & $\begin{array}{l}\text { Average } \\
\text { payoff }\end{array}$ & -1.00 & $(0.00)$ & -0.62 & $(0.09)$ & -0.29 & $(0.46)$ \\
\hline 3) & $\begin{array}{l}\text { \# action } \\
\text { changes }\end{array}$ & $\begin{array}{l}\text { \# different } \\
\text { networks }\end{array}$ & 1.00 & $(0.00)$ & 1.00 & $(0.00)$ & 0.93 & $(0.00)$ \\
\hline 4) & $\begin{array}{l}\text { Time spent } \\
\text { in ps-stars }\end{array}$ & $\begin{array}{l}\text { Average } \\
\text { payoff }\end{array}$ & 0.76 & $(0.02)$ & 0.83 & $(0.01)$ & 0.91 & $(0.00)$ \\
\hline
\end{tabular}

Table 4: Spearman rank correlation coefficients $r_{s}$

We find positive correlations in each of the Treatments I-III between

- the number of action changes and the number of formed networks,

- the time spent in ps-stars and the average payoff.

These correlations are not surprising. When a group is characterized by a large number of action changes, i.e. if it is active in changing links, it may have a better chance of forming new networks than an inactive group with a small number of action changes. This is reflected in relationship 3. Relationship 4 easily follows from the fact that psstars are efficient states. Groups which stay in ps-stars for a long time will achieve a higher average payoff per player than other groups.

We find two negative correlations in each of the Treatments I-III between

- the number of action changes and the time spent in ps-stars,

- the number of action changes and the average payoff.

The first relationship is plausible since more changes of links diminish the time spent in ps-stars. Therefore, we expect that groups with a larger number of action changes show a tendency to spend less time in ps-stars. This is reflected in relationship 1. Relationship 2 is more difficult to understand. It expresses a fact which meanwhile has been observed in other experiments of quite different types. High activity levels of the subjects in those 
experiments are often connected with low payoffs (see, for example Berninghaus et al. 1999, Chmura and Pitz 2006, Selten, Schreckenberg, Pitz, Chmura and Kube 2006). According to these results, to be successful in earning monetary payoffs one should not be "too active." At first glance, this seems to be surprising. Concerning our experiment on network formation it may be explained by the fact that players have to solve a difficult coordination problem. A high intensity of individual action changes or a high degree of experimenting with links seems to make it more difficult for the players to agree on the same center player and, therefore, may prolong the time until players reach efficient networks. This relationship is significant at a 5\%-level for Treatment I. For Treatment II it is only significant at a 10\%-level, and for Treatment III this interrelationship is not significant.

\subsection{Differences between treatments}

In this section we check if there are significant differences between the treatments with respect to some selected performance variables (see Table 5). For this purpose, we apply standard non-parametric tests to our data (Kruskal-Wallis and Mann-Whitney rank sum test; significant differences based on a $5 \%$ level are presented in boldface in Table 5).

\begin{tabular}{|c|c|c|c|c|}
\hline \multirow[t]{2}{*}{$\begin{array}{l}\text { Performance } \\
\text { variables }\end{array}$} & \multirow{2}{*}{$\begin{array}{c}\text { Kruskal-Wallis: } \\
\text { Test statistic ( } p \text {-value) } \\
\text { Treatment I,II,III }\end{array}$} & \multicolumn{3}{|c|}{$\begin{array}{c}\text { Mann-Whitney: } \\
\text { Test statistic ( } p \text {-value) }\end{array}$} \\
\hline & & Treat. I,II & Treat. I,III & Treat. II,III \\
\hline$\#$ action changes & $0.74(0.69)$ & $62(0.57)$ & $66(0.88)$ & $76(0.44)$ \\
\hline $\begin{array}{l}\text { \# different center } \\
\text { players in ps-stars }\end{array}$ & $6.59(0.04)$ & $44.5(0.01)$ & $51.5(0.08)$ & $68.5(0.96)$ \\
\hline Time in ps-stars & $0.74(0.69)$ & $65(0.80)$ & $69(0.96)$ & $78(0.33)$ \\
\hline $\begin{array}{l}\text { Time until first } \\
\text { ps-star reached [\%] }\end{array}$ & $9.32(0.01)$ & $82(0.001)$ & $73(0.054)$ & $58.5(0.33)$ \\
\hline \multirow[t]{2}{*}{ Average payoff } & $13.90(0.00)$ & $93(0.01)$ & $100(0.00)$ & $83(0.13)$ \\
\hline & Treatment II,III,IV & Treat. II,III & Treat. II,IV & Treat. III,IV \\
\hline $\begin{array}{l}\text { Time spent in empty } \\
\text { network }\end{array}$ & $17.01(0.00)$ & $36(0.00)$ & $55(0.002)$ & $52(0.01)$ \\
\hline
\end{tabular}

Table 5: Treatment differences in performance variables

The Kruskal-Wallis test shows that there exist significant differences between treatments in the number of different ps-stars reached, in the time until a ps-star is reached for the first time, and the average payoffs. The Mann-Whitney tests give more information by pairwise comparison of treatments. Considering the number of different ps-stars reached, we see that the differences between Treatments II and III are not significant but between Treatment I and Treatment II .

Although we compensate the difference in potential earnings in the different treatments (for details see Section 3), the Kruskal-Wallis test reveals significant differences. 
These are caused by the significant differences between Treatment I and the other two treatments. In contrast to Treatment I, we have the empty network as an additional strict Nash network in Treatments II and III. We observe that the subjects in Treatment III actually choose the empty network very often. In Treatment II the empty network plays a minor role and in Treatment I it is never observed. This is certainly an additional determinant that creates the average payoff differences between the treatments. We design our experiment such that the fixed-payment part of the maximum possible earnings grows with increasing link costs and we observe that the resulting average payoff decreases, which indicates that the groups become less efficient from one treatment to the next.

\subsection{Inequity aversion as a plausible motive that drives our re- sults}

In our particular experimental design, the ps-star is the only strict Nash network for $a>c$. Falk and Kosfeld use the theoretical model of Bala and Goyal in which the center-sponsored star is the only strict Nash network for this parameter constellation. In a center-sponsored star the only active player (i.e. the center player) reduces his own payoff by $a-c$ if he severs one link to a periphery player. In our design, an active periphery player reduces his payoff from $(n-1) a-c$ to zero by severing the link to the center player. Thus, in our design the costs of deviation are higher. Moreover, the payoff difference between the center and a periphery player in a center-sponsored star is greater than in a ps-star. In this context, Falk and Kosfeld conjecture that inequity aversion might have prevented subjects from forming a center-sponsored star, i.e. the only strict Nash equilibrium.

We observe that groups form ps-stars and consecutively exchange the center player. They leave the strict Nash network (i.e. a ps-star) in order to form a new one with a different center player. What might be the reasons for this behavior? In our design, center players are subsidized by the periphery players. Even if the instantaneous payoff difference between center and periphery player in a ps-star is not that large, accumulated payoff differences after 30 minutes show a considerable difference. In order to establish payoff equalization in the long run, groups have to exchange the center player which is easier to achieve in our continuous time framework than in a discrete time setting. Therefore, we think that the principle of inequity aversion is an important motive that drives this observation.

\section{Concluding remarks}

By appropriately modifying the original model of network formation we observe that groups prefer to stay in strict Nash networks for some time and, moreover substitute the respective center player to reach ps-stars with different center players. Similar systems of rotation have been observed by anthropologists in societies in New-Guinea (Rubel and Rosman 1978, Rappaport 1968). In the Maring society, for example, each clan serves 
as a host for a big feast to neighbor clans. At the feast, many pigs and other food are eaten. People use this opportunity to exchange information and goods. The whole feast is very costly to the host who can be regarded as the center player in a center-sponsored star network whereas the guests are the periphery players. The clans have the obligation to reciprocate, which results in the rotation of center players in the stars. Each clan has to become host at one time. Although our model does not perfectly match the anthropologists' stories since we have ps-stars, it is easy to transfer the main aspects of these observations to our framework. Rotation of center players in center-sponsored stars compensates the center players for bearing all link costs. The rotation of center players in ps-stars pushes each group member into the same privileged situation in which her connections are subsidized by the other group members. Both phenomena can be explained by inequity aversion.

\section{References}

Bala, V., \& Goyal, S. (2000). A non-cooperative model of network formation. Econometrica, 68(5), 1181-1229.

Berninghaus, S. K., \& Ehrhart, K.-M. (1998). Time horizon and equilibrium selection in tacit coordination games: Experimental results. Journal of Economic Behavior and Organization, 37, 231-248.

Berninghaus, S. K., Ehrhart, K.-M., \& Keser, C. (1999). Continuous-time strategy selection in linear population games. Experimental Economics, 2, 41-57.

Berninghaus, S. K., Ehrhart, K.-M., \& Ott, M. (2005). A network experiment in continuous time: The influence of link costs. SFB504 Discussion Paper Series No. 05-02, University of Mannheim.

http://www.sfb504.uni-mannheim.de/publications/dp05-02.pdf

Berninghaus, S. K., Ehrhart, K.-M., Ott, M., \& Vogt, B. (2004). Searching for "stars" - recent experimental results on network formation -. SFB504 Discussion Paper Series No. 04-34, University of Mannheim. http://www.sfb504.uni-mannheim.de/publications/dp04-34.pdf

Callander, S., \& Plott, C. R. (2005). Principles of network development and evolution: An experimental study. Journal of Public Economics, 89, 1469-1495.

Chmura, T., \& Pitz, T. (2006). Successful strategies in repeated minority games. Physica A, 363, 477-480.

Deck, C., \& Johnson, C. (2004). Link bidding in laboratory networks. Review of Economic Design, 8, 359-372.

Falk, A., \& Kosfeld, M. (2003). It's all about connections: Evidence on network formation. IEW Working paper No. 146, University of Zrich. 
Friedkin, N. E. (1983). Horizons of observability and limits of informal control in organizations. Social Forces, 62, 54-77.

Goeree, J. K., Riedl, A., \& Ule, A. (2005). In search of stars: Network formation among heterogeneous agents. Working paper.

Goyal, S. (2005). Learning in networks. In G. Demange and M. Wooders, editors, Group Formation in Economics; Networks, Clubs and Coalitions, chapter 4. Cambridge University Press, Cambridge U.K.

Granovetter, M. (1995). Getting A Job: A Study of Contacts and Careers. University of Chicago Press, 2nd edition.

Jackson, M. O. (2005). A survey of models of network formation: Stability and effciency. In G. Demange and M. Wooders, editors, Group Formation in Economics; Networks, Clubs and Coalitions, chapter 4. Cambridge University Press, Cambridge U.K.

Kosfeld, M. (2004). Economic networks in the laboratory: A survey. Review of Network Economics, 3, 20-41.

McBride, M. (2006). Imperfect monitoring in communication networks. Journal of Economic Theory, 126, 97-119.

Oberschelp, W. (1967). Kombinatorische Anzahlbestimmungen in Relationen. Mathematische Annalen, 174, 53-78.

Rappaport, R. A. (1968). Pigs for the Ancestors. Yale University Press.

Rubel, P. G., \& Rosman, A. (1978). Your Own Pigs You May Not Eat. The University of Chicago Press.

Selten, R., Schreckenberg, M., Pitz, T., Chmura, T., \& Kube, S. (2006). Commuters route choice behaviour. Games and Economic Behavior. Forthcoming. 